\title{
Difficult Lumbar Puncture: Pitfalls and Tips from the Trenches
}

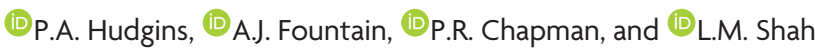

\begin{abstract}
SUMMARY: Lumbar puncture has, for many years, been the responsibility of the internal medicine physician or the neurologist. As more patients have undergone spine surgery and with the current increase in body mass index of the general population, the radiologist has been consulted with increasing frequency to perform lumbar puncture with fluoroscopic guidance. Radiology, in fact, is now the dominant overall provider of lumbar puncture procedures. The procedure is more difficult when the needle length increases, and if fluoroscopy is used, landmarks are more difficult to visualize with increasing subcutaneous fat. Our goal with this review was to describe our techniques for lumbar puncture in the difficult patient, with emphasis on using fluoroscopy in the obese patient and to suggest maneuvers that might make the procedure easier. Combining our experience from performing these procedures on an obese population, we would like to share our tips, especially with trainees early in their career.
\end{abstract}

ABBREVIATIONS: $B M I=$ body mass index; IIH = idiopathic intracranial hypertension; $\mathrm{LP}=$ lumbar puncture

umbar puncture (LP) has, for many years, been the responsibility of the internal medicine physician or the neurologist. As more patients have undergone spine surgery and with the current increase in body mass index (BMI) of the general population, the radiologist has been consulted with increasing frequency to perform the LP fluoroscopic guidance. ${ }^{1}$

Current estimates from the Centers for Disease Control and Prevention are that more than one-third (34.9\% or 78.6 million) of adults in the United States are obese. ${ }^{2}$ BMI is calculated as weight in kilograms divided by the square of the height in meters, and obesity is defined as a BMI of $>30$. In our practice, patients often weigh $>136$ $\mathrm{kg}$, with a BMI of $>50$. The financial impact of obesity on the health care system is well-known, because this chronic condition contributes to the development of diabetes, cardiovascular issues, and, now increasingly recognized, idiopathic intracranial hypertension (IIH), previously termed "pseudotumor cerebri." 3,4

The impact of obesity on the radiology practice is now being recognized. Patients are not able to fit on imaging equipment,

From the Department of Radiology and Imaging Sciences (P.A.H., A.J.F.), Division of Neuroradiology, Emory University School of Medicine, Atlanta, Georgia; Department of Radiology (P.R.C.), University of Alabama, Tuscaloosa, Alabama; and Department of Radiology and Imaging Sciences (L.M.S.), University of Utah, Salt Lake City, Utah.

Please address correspondence to Patricia A. Hudgins, MD, FACR, Emory University Hospital, 1364 Clifton Rd NE, Atlanta, GA 30322; e-mail: phudgin@emory.edu; @phudge54

- Indicates open access to non-subscribers at www.ajnr.org

http://dx.doi.org/10.3174/ajnr.A5128 including procedural equipment, designed for the "averagesized" patient. The obese patient who needs CSF sampling presents multiple potential difficulties. Equipment has weight limits above which table function is not assured, and the fluoroscopy table may not tilt. Increasing BMI has been shown to result in a longer fluoroscopy time for LP access. ${ }^{5} \mathrm{LP}$ is more difficult when the needle length increases, and if fluoroscopy is used, landmarks are more difficult to visualize with increasing subcutaneous fat. Conventional imaging parameters are not sufficient to penetrate extra layers of patient fat; the density properties of human tissue become problematic in patients weighing $\geq 113 \mathrm{~kg} .{ }^{6,7}$

Previously, LPs were typically performed without image guidance; however, radiology is now the dominant overall provider of LP procedures. ${ }^{1}$ Image guidance is often requested when there is postoperative hardware and/or osseous fusion, extensive degenerative change, or scoliosis ${ }^{8}$; after multiple failed attempts without imaging; and with the inability to identify or palpate spinous processes or the iliac crest, osseous landmarks routinely used to plan LP, such as in the obese patient. One of the most common indications for LP in the obese patient is confirming or treating $\mathrm{IIH}$, a known complication of obesity. For diagnosis alone, CSF pressure measurements may be all that are required. However, for the symptomatic patient, especially with headache or fluctuating vision loss, removing CSF is therapeutic.

Our goal in this review was to describe our techniques for LP in the difficult patient, with emphasis on the obese patient, and suggest maneuvers that might make the procedures easier. Because fluoroscopically guided LP is a common procedure performed by 


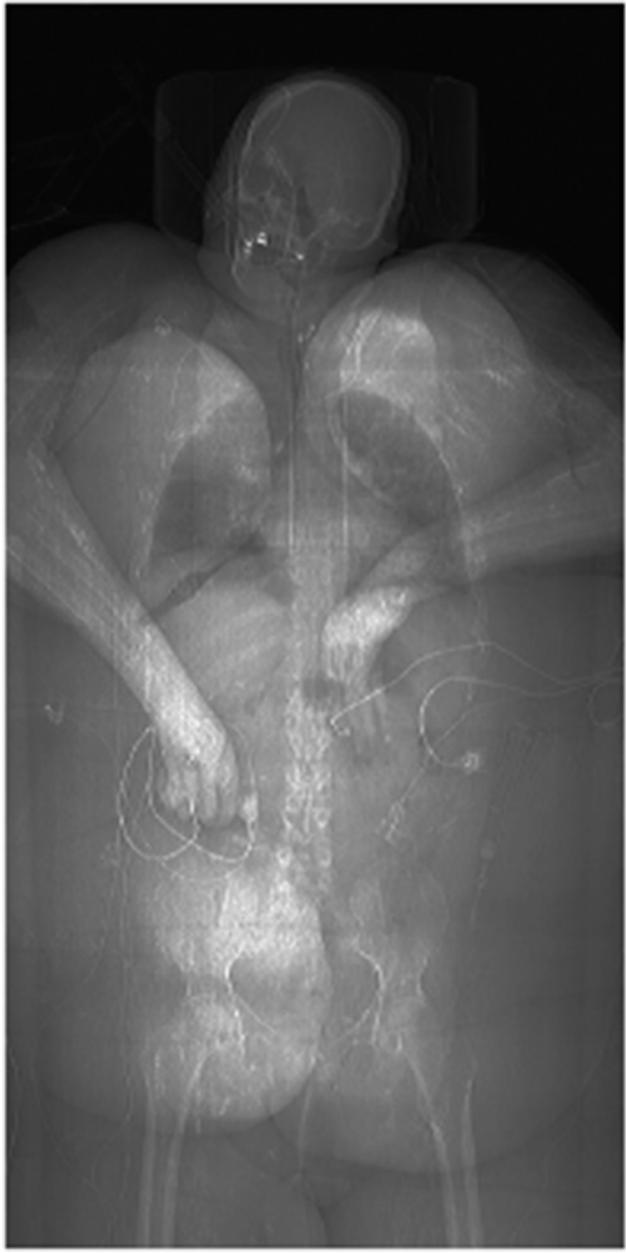

FIG 1. Adult woman, $\mathrm{BMI} 55$, in a serious motor vehicle collision. Scout CT scan for chest, abdomen, and pelvis shows the hands in the midline, loosely tied with tape so the patient could fit through the CT bore.

the neuroradiologist, experienced physicians likely have their own tricks. Combining our experience in performing these procedures on an obese population with those of other interventionalists with similar challenges, we would like to share our tips, especially with trainees early in their careers.

\section{Fluoroscopy or CT?}

Most LPs in our practice are performed with fluoroscopy because it is faster and does not tie up a CT scanner that might be needed for critical inpatients or those in the emergency department. The table load weight limit indicates the z-axis accuracy as the patient goes through the scanner, ensuring the diagnostic quality of the image. ${ }^{9}$ However, if the weight limit is exceeded, the table may bend or break with possible injury to the patient. CT scanners have variable table weight limits, and vendors now offer bariatric tables to accommodate larger patients. Furthermore, the body habitus of many of our patients does not allow them to fit in the scanner with the needle in place. Thus, the usable portion of the anteroposterior diameter is important to know, not just the gantry aperture. ${ }^{9}$ We have occasionally had to tie or tape patients' hands together and loosely bind redundant skin folds to just get a diagnostic CT scan (Fig 1). Additionally, the CT table usually

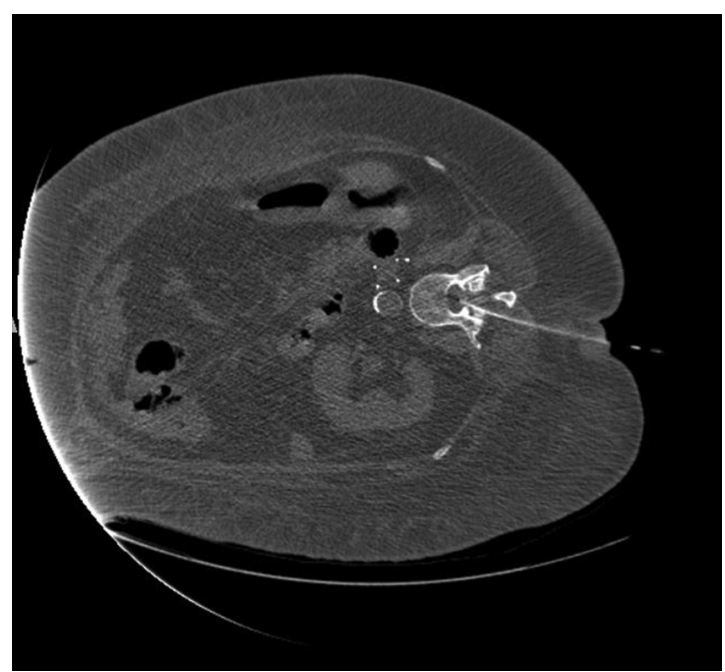

FIG 2. Adult patient, $B M I 49$, with bowel incarceration requiring an operation, now with altered mental status. LP was requested to exclude meningitis. The patient is in the right lateral decubitus position so that the respiratory technologist can control the tracheostomy and airway. Note the tip of the needle in the mid-spinal canal.

cannot tilt; this maneuver facilitates the flow of CSF. Using a fluoroscopic C-arm is the ideal method because the obese patient often cannot lie prone or even slightly oblique. However, our $\mathrm{C}$-arm fluoroscopic machines are usually scheduled for interventional use, and we initially attempt the LP with conventional fluoroscopy most of the time. It is obviously essential that the fluoroscopy tower clear the patient and the needle; otherwise, a C-arm is essential.

Radiation doses from fluoroscopy versus CT are reportedly comparable for LPs performed in obese patients. ${ }^{10}$ Data from phantom studies indicate that obese patients receive higher radiation doses from CT and radiography than nonobese patients. ${ }^{11,12}$ For severely ill or intubated patients in the hospital, obese or not, CT or a biplane image intensifier is invaluable, with the patient in the lateral decubitus position (Fig 2). This allows greater comfort, less respiratory motion, better airway control by the respiratory therapist, and assurance that the patient will fit in the gantry with the LP needle in position.

\section{Planning the Examination}

We almost always have prior brain imaging available, ideally within 30 days of the LP, to be sure there is no mass, hydrocephalus, or mass effect that may result in herniation when the spinal pressure is lowered by removing CSF. A recent physical examination with normal findings documented by a neurosurgeon or neurologist can also suffice. Especially if there has been a prior lumbar spine operation, plain radiographs or postoperative cross-sectional imaging is essential. This preprocedural image review helps determine the best level for the procedure and can show surgical complications such as hardware failure, arachnoiditis (Fig 3), or spinal infection. A scar from a prior operation, if mature, is generally not sensitive, but lidocaine is still used subcutaneously. A new postoperative scar tends to be exquisitely sensitive, and if possible, we avoid these levels. Any bone window, whether a normal space or as a result of prior surgery, potentially can be used to gain access.

AJNR Am J Neuroradiol 38:1276-83 Jul 2017 www.ajnr.org 


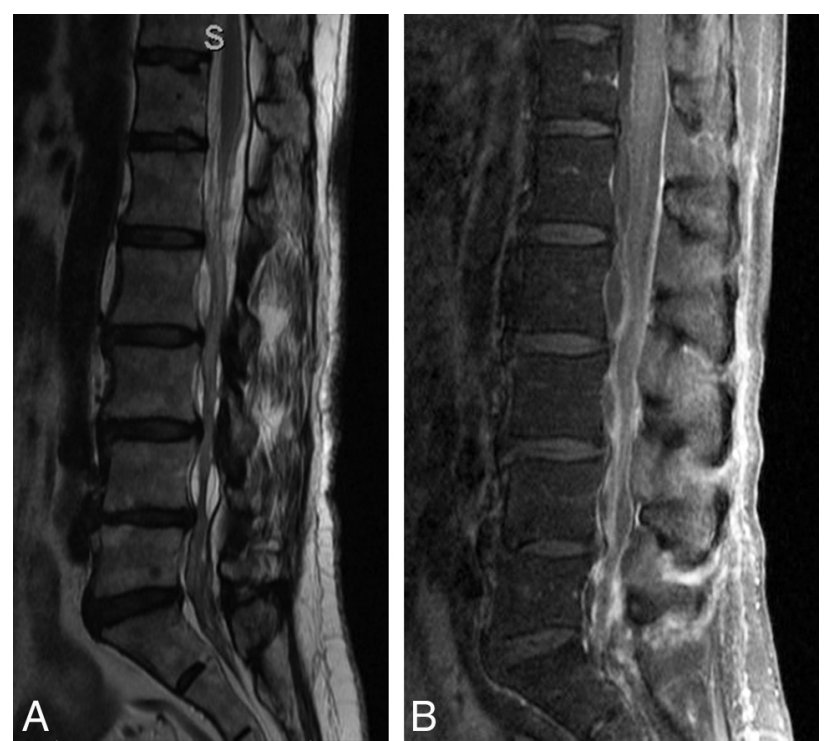

FIG 3. Adult patient with multiple epidural lumbar steroid injections, now recalcitrant to more injections. The patient experienced severe pain during an attempted LP. Note severe arachnoiditis. A, T2 sagittal MR image shows marked clumping of the cauda equina in the central thecal sac, but it also adhered to the posterior dural wall. The conus appears irregular, also from arachnoiditis. $B, T 1$ sagittal, postgadolinium image with fat saturation shows diffuse enhancement of the nerve roots and meninges. Arachnoiditis does not always enhance. Because meningitis could have a similar appearance, CSF must be obtained to exclude infection, despite the arachnoiditis.

If there is prior lumbar spine MR imaging or abdominal CT imaging, the distance between skin and thecal sac can be measured and use of a longer needle can be planned. Measurement is made typically from the left parasagittal region, the expected site of the proposed LP. The depth of the thecal sac is variable over the length of the lumbar spine but often decreases from inferior to superior. For example, in a given patient, a $3.5-\mathrm{cm}$ needle may be sufficient at the L2-3 level but may be too short to reach at the L5-S1 level. A pillow beneath the hips may decrease the lumbar lordosis and slightly "stretch out" the subcutaneous fat. If there is no imaging available, we palpate the lumbar spinous processes. If they cannot be palpated, it is likely that the standard $3.5-\mathrm{cm}$ needle will not be long enough. This is a relatively crude measurement, but in our experience, it works well. Nayate et $\mathrm{al}^{13}$ have proposed a formula [Skin-Canal Distance (inches) $=0.077 \times$ $\mathrm{BMI}+0.88]$ to predict the appropriate needle length in oblique interlaminar-approach LP using the BMI. In some obese patients, there is a large "buttock shelf" with almost a right angle with the back (Fig 4). Occasionally, we may need a second person to gently push this large shelf inferiorly.

Review of prior spine imaging helps avoid levels of spinal stenosis, where the thecal sac is narrowed and nerve roots are clumped. Increased fat in the epidural space, especially at L4 or lower, should also be avoided (Fig 5). At a stenotic level, the patient will likely experience radicular pain with the procedure, and CSF return will be slow or nonexistent. Therefore, it will be beneficial to approach the level above the stenosis to access the subarachnoid space.
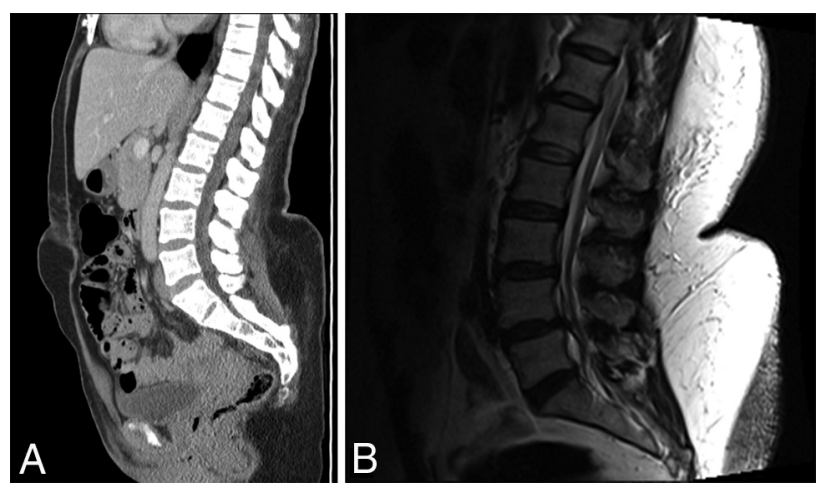

FIG 4. Adult female patients with morbid obesity and large fat shelf on the lower lumbar back. A, Sagittal reformation from a noncontrast abdominal and pelvis CT shows the deformity of the back, even with the patient supine. $B$, Sagittal T2 MR image in another patient with marked subcutaneous fat in the lower thoracic and lumbar back. Note an abrupt increase in fat thickness at the L3 level, which makes LP difficult.

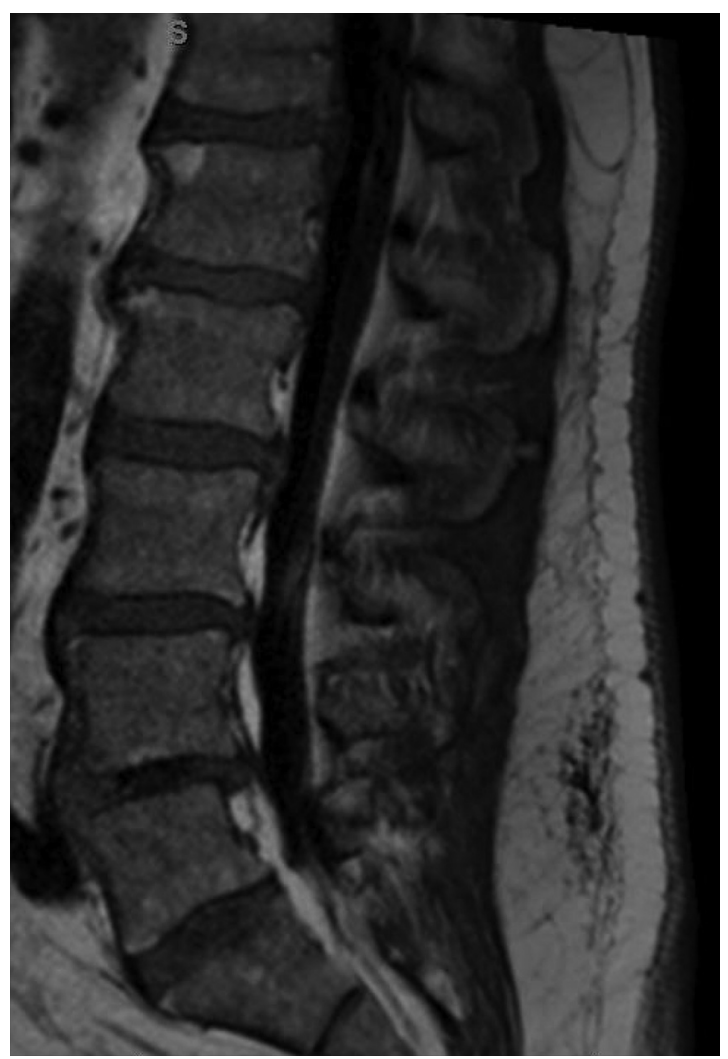

FIG 5. A man with epidural lipomatosis. $T 7$ sagittal MR image shows high-signal-intensity fat around the thecal sac, with marked narrowing at L5.

\section{Patient Experience and Comfort}

We have all heard about negative experiences with LP. The goal of the radiologist should be to provide an LP procedure with as little anxiety, discomfort, and pain as possible. While obtaining consent, the radiologist should be calm, relaxed, and unhurried and offer positive reassurance that the goal is to provide a pain-free experience. The details of the procedure and the risks and benefits should be conveyed in layman's terms. The small risk of spinal headache, infection, and nerve injury is important to communicate but with reassurance that the risks are low. If the patient 


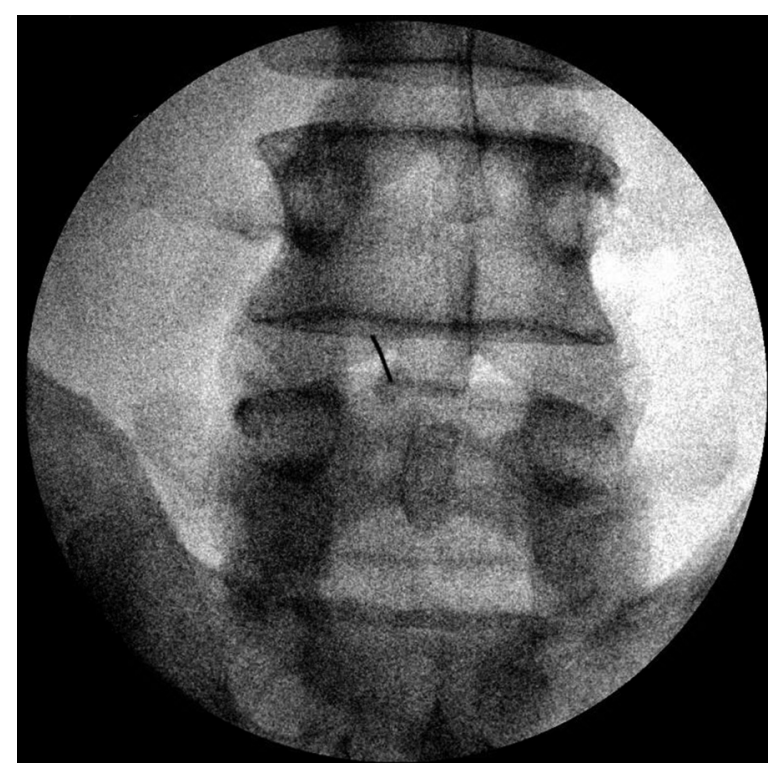

FIG 6. Spot fluoroscopic image showing a 23-ga needle near the lamina for deep anesthesia. It is critical that the lidocaine not be injected into the spinal canal or thecal sac. We never use the anesthesia needle in the thecal sac.

experiences what is likely a low-pressure headache, he or she may be advised to call the radiology department and speak with a neuroradiologist. We discuss the headache with the patient, and advise increasing caffeine intake and bedrest for at least 2 additional days before treatment by a blood patch is considered. Low-pressure headache treatment is institutional-dependent.

The patient should be prone or prone oblique on the fluoroscopy table, with only a thin pillow under the head, arms by the side, and a small bolster at the abdominal level if there is a lordosis. With the patient in the oblique position, we have the top leg, usually the right leg, bent. Local anesthesia is important. While some providers minimize the effects of local anesthesia and may claim that the lidocaine injection is more painful than the lumbar puncture itself, we consider it essential for patient comfort. Lidocaine $\mathrm{HCl} 1 \%$ concentration itself can be painful, but the pain can be reduced by a slower, gentler injection to create a cutaneous and subcutaneous wheal. After we create a skin wheal, the 25-ga needle is advanced through the subcutaneous tissues and several milliliters of lidocaine is injected. We have used $1 \mathrm{~mL}$ of bicarbonate solution mixed with $4-5 \mathrm{~mL}$ of lidocaine to mitigate the initial sting.

One trick is to use a 25 -ga $3.5-\mathrm{cm}$ spinal needle in all patients to deliver anesthesia to the deeper tissues and periosteum (if the needle reaches it). Unlike larger needles, the 25 -ga needle can be advanced through the tissues with minimal or no discomfort for the patient. After we numb the skin, the 25-ga needle is advanced through the subcutaneous tissues to the fascia of the paraspinal muscle, the stylet is removed, and several milliliters of lidocaine is injected. The needle can be advanced with the syringe still attached, injecting into the muscle. With fluoroscopy to be sure the needle is not intraspinal, the needle can even be directed to the lamina (either above or below the interlaminar space) (Fig 6). Thus, both the periosteum and the distal medial branches of the dorsal ramus can be numbed to provide anesthesia to the deep soft tissues and prevent referred radicular pain. This maneuver also determines the depth necessary for the LP.

We recommend never injecting lidocaine through the LP needle because injecting lidocaine into the thecal sac can be dangerous, potentially resulting in motor weakness or arachnoiditis. Also, lidocaine in the needle or hub can make identification of CSF more difficult.

While you advance the spinal needle, it is helpful to secure the hub and brace your hands against the patient to guard against sudden patient movement, which may inadvertently advance the needle. It is critical to recognize that the greatest pain can be produced by advancing the needle into an intradural spinal nerve and producing a lancinating pain to the lower extremity, hip, buttock, or groin. When the needle is at or within the ligamentum flavum, we remind the patient that the needle is approaching nerves that have not been anesthetized and ask the patient to speak up if there is radicular pain (focal sharp pain down $1 \mathrm{leg}$ ). The needle is then advanced extremely slowly and gently at this point. If radicular pain is felt, the operator should immediately stop the needle, withdraw it approximately $1 \mathrm{~mm}$, and check for fluid. Remember that $1 \mathrm{~mm}$ of movement can be the difference between experiencing pain or not. Pain usually abates within seconds once the needle is off the nerve. If the patient continues to have severe radicular pain, a different approach needs to be taken altogether.

During the examination, some patients, in our experience young males in particular, may have a vasovagal response. The radiologist should be aware of these signs: increased anxiety, paleness, sweating, or comments like, "I feel really hot" or "I feel like I am going to throw up." Typically, we tell the patient that we are going to break for few moments, put the table in the Trendelenburg position, and offer a cold washcloth to the face. After a minute or so, the procedure can be completed without further progression of the response.

The spinal headache is the most common complication following LP. In our experience, the LP headache occurs far less often in patients with documented IIH. Typically, the headache will be at maximum 1-2 days after the procedure and will take 1-2 days to fully resolve. Most headaches occurring after a single needle stick will resolve spontaneously without a blood patch. Patients are advised to restrict strenuous or athletic activity for 24 hours following the procedure to prevent the headaches. While some authors have suggested the use of an "atraumatic" needle such as the Whitacre needle, we think that the most effective way to prevent postprocedural headache is by limiting the procedure to a single puncture of the posterior thecal sac. Replacing the stylet before removal of the needle may decrease the incidence of postlumbar puncture headache. ${ }^{14,15}$ We have patients lie in the recumbent position for approximately 30 minutes after the LP; however, this has not been shown to be effective in preventing postprocedural headache. ${ }^{16}$ If the headache is still present $\geq 4$ days after the LP and there is no improvement, we consider referral for an epidural blood patch.

\section{Technique}

We routinely use either the prone or prone oblique position and aim for the interlaminar location or "behind the Scotty dog's 


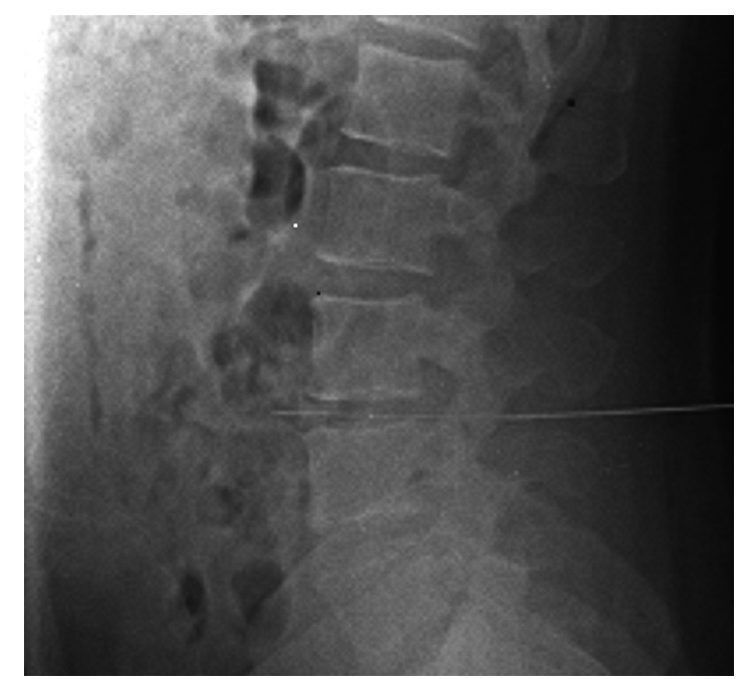

FIG 7. Cross-table lateral film after failure to obtain CSF, thought to be a dry tap. Note the LP approach at the L4 -5 disc space and the needle extended through the disc into the retroperitoneum. At this point, the stylet was removed and no blood was returned. The needle was withdrawn at 5-mm increments, each time checking for arterial or venous blood return. There were no complications, but the diagnostic LP was cancelled until the following day.
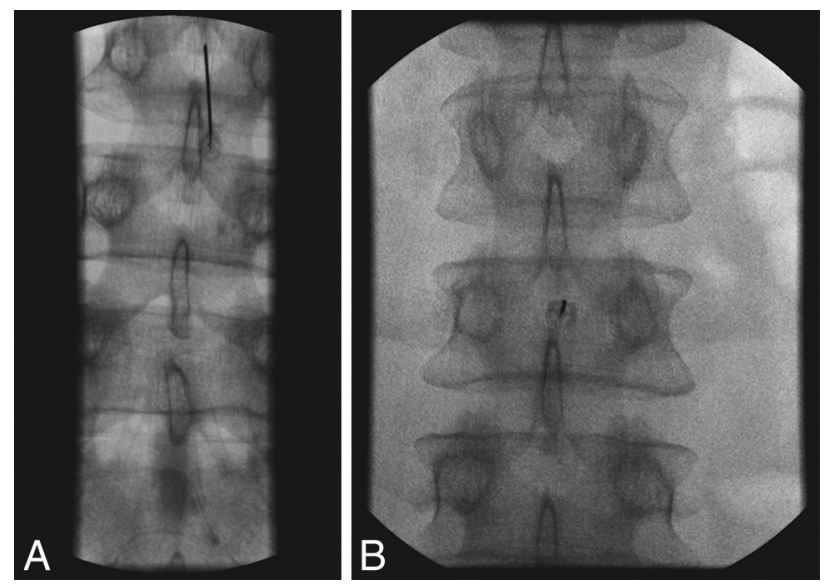

FIG 8. Fluoroscopic spot films obtained during LP, showing parallax artifacts. $A$, Note that on this image, the needle is not centered and the tip appears to abut the inferior spinous process. $B$, The same procedure with no needle adjustment, but now the tip is centered in the image. CSF flow was normal.

neck," avoiding the disc space level. Because an LP needle can easily extend through the disc itself, terminating in the prevertebral location or retroperitoneum (Fig 7), we avoid the disc space level. If there is inadvertent extension through a disc with the spinal needle, a rare event, we have found that replacing the stylet and removing the needle at $1 / 2$-cm intervals, with frequent checks for blood return, is a method to deal with this complication. We have never had venous or arterial blood return, but if that occurred, we would plan to document the blood return, attempt to differentiate venous from arterial blood, and consider a CT angiogram/venogram of the aorta and inferior vena cava after terminating the unsuccessful LP. It is critical that when using fluoroscopy, the needle be centered in the image to avoid parallax artifacts, an effect distinct to radiographs (Fig 8). Parallax is a difference in the apparent position of an object along 2 different lines of sight. ${ }^{17}$

Experienced radiologists are often comfortable with a 25 -ga spinal needle, but if a longer needle is needed, a 25 -ga needle is more difficult to steer. Our standard needle is $22 \mathrm{ga}$. For extremely obese patients, we often use a 3.5-inch 18-ga introducer, but this larger gauge should not enter the thecal sac. ${ }^{18}$ The introducer is advanced using the same landmarks used in the LP and is hubbed. Although the introducer needle is easy to steer, it is important to have a properly planned trajectory because it can be difficult to correct the path with this larger gauge coaxial needle. The needle is much easier to steer through the introducer. The standard spinal needle is $8.8 \mathrm{~cm}$ (3.5 inches). Needles are also available at 13-, 14-, 15-, and 18-cm or even longer lengths.

The 22-ga 19-cm spinal needle is then advanced through the introducer. The needle hub has a notch, which is on the bevel side. Therefore, the side opposite the notch is the "sharp" side. The needle will go toward this sharper side and away from the notch. Therefore, if you are steering the needle, the notch should be opposite the direction you are attempting to steer. Some introducer needles may have the bevel on the opposite side of the notch on the hub. Turning the needle $1 / 4$ turn every $1 \mathrm{~cm}$ or so helps direct the needle in a straight line. The introducer will help with needle position, but it is still important to check periodically with the fluoroscopy for an adequate trajectory. Even with the longer spinal needle, the "pop" through the dura can often be felt. The bevel should be directed cephalad, away from the dura/arachnoid and perpendicular to the course of the nerves, to improve exposure to the fluid, particularly if there is slow flow.

The obese patient usually cannot roll on the table for an opening pressure in the lateral decubitus position, so we obtain the pressure in the prone or prone oblique position, adding the needle length to the final pressure measurement. ${ }^{19}$ The normal CSF pressure range is $6-20 \mathrm{~cm} \mathrm{H}_{2} \mathrm{O}$ in adults and up to $25 \mathrm{~cm} \mathrm{H}_{2} 0$ in obese patients. ${ }^{20}$ An opening pressure of $>25 \mathrm{~cm} \mathrm{H}_{2} 0$ is diagnostic of $\mathrm{IIH}$, in the correct clinical setting. ${ }^{21}$

For the diagnostic LP, how much CSF to remove and put in each tube varies on the basis of what has been requested by the referring physician or provider. We routinely attach short tubing to the needle and use a $10-\mathrm{mL}$ syringe after the seal has been broken, to gently remove CSF. The suction is applied very gently, for just 2-3 seconds, then released to avoid affecting a nerve root. This process is then repeated as long as CSF return is present. One of the authors uses a unique technique that allows air release into the tube-hub interface (Fig 9). The connection between the tubing and hub is loose and not airtight, thus preventing oversuction of fluid. For the standard diagnostic LP, CSF is collected in 4 tubes for a total of $8-15 \mathrm{~mL} .{ }^{22}$ If leptomeningeal carcinomatosis is suspected, we get the usual specimen for the initial cell count, protein and glucose levels, and culture; then, fluid for cytology can be placed either in a black-top tube or in one of the standard LP tubes on the tray. We always send the last $1-2 \mathrm{~mL}$ for the final cell count.

Flow cytometry in lymphoma or leukemia is unpredictable. The laboratory needs "viable" cells to perform the test, and it is impossible to know how much CSF is truly necessary. It can be done with $3 \mathrm{~mL}$ of fluid, depending on the viable cell population. 


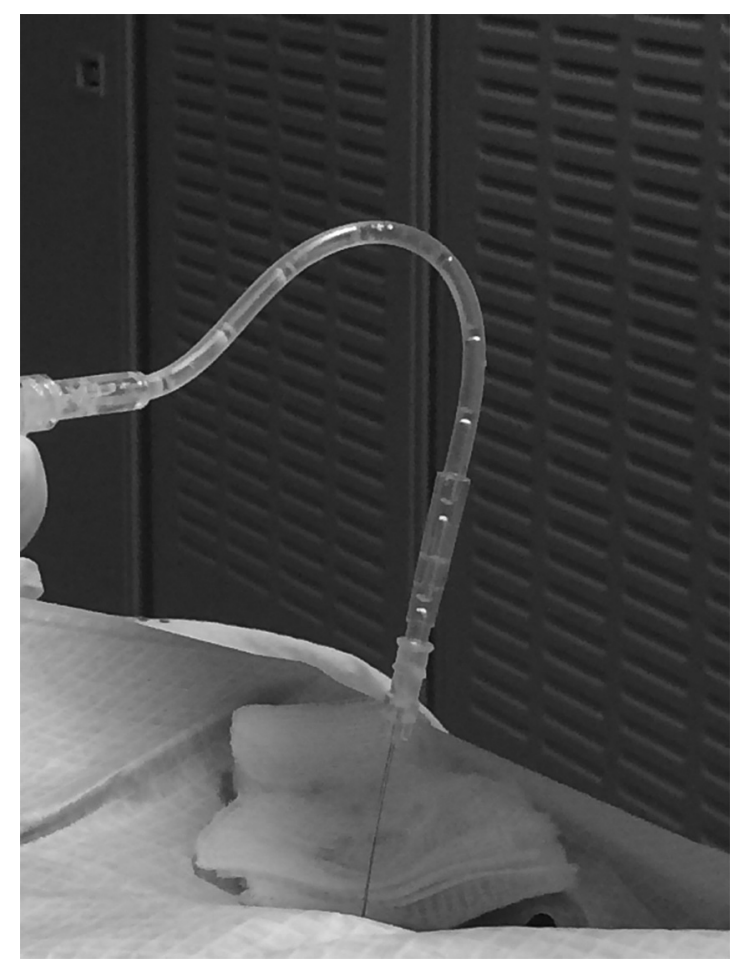

FIG 9. Bubble technique to avoid sucking nerve roots into the spinal needle. The short tubing is literally lying on the LP needle hub so that there is no manipulation of the needle. Both air and CSF fill the tubing and the syringe. With this technique, we have never had nerve root pain reported, and CSF can be easily aspirated.

However, $10 \mathrm{~mL}$ of fluid might be inadequate at other times if the load of abnormal cells in the CSF is small. We try to obtain a separate vial of $6-10 \mathrm{~mL}$ when flow cytometry is requested. Flow cytometry should be distinguished from cytology.

\section{Therapeutic LP: How Much CSF to Remove?}

The therapeutic LP generally implies removal of CSF to both confirm the diagnosis of IIH and treat the symptoms of intracranial hypertension, which in some institutions can mean removal of $>30 \mathrm{~mL}$ of CSF. When LP is performed to remove CSF, we ask the patient about a headache or visual obscurations before the study and symptom changes during the procedure and then ask again once CSF has been removed. In our experience, a high-volume tap is rarely needed, and there is a risk of low-pressure headaches after the LP. Most patients with IIH do well with a standard amount of CSF removed (15-20 mL). This is likely because the dural defect continues to leak after the tap. In addition, the high intracranial pressure in IIH results in distal transverse venous sinus narrowing; removing CSF decreases the intracranial pressure and reduces venous sinus narrowing, improving venous return with a resulting decrease in symptoms. The opening and closing pressures, the amount of CSF removed, and symptom resolution are documented in the final dictation. The closing pressure is only obtained when performing a therapeutic tap.

\section{Dry Tap}

"Dry tap" is defined as an LP with confirmed needle location in the thecal sac, but no egress of CSF. Confirmation of the needle position is by a cross-table lateral radiograph. In our experience, the dry tap most commonly occurs in the hospitalized patient who has not been eating or drinking and is relatively dehydrated. Before any LP in the hospitalized patient, we ask the team to hydrate the patient, ideally with intravenous fluids. For outpatients, we use no sedating medications, and patients are not NPO and are instructed to drink fluids liberally. Anecdotally, we have noticed that the elderly patient will often have a dry tap and very low CSF pressure, and CSF flow may be very slow. Dry taps can occur in severe spinal stenosis, including thecal sac narrowing from epidural lipomatosis (Fig 5) or arachnoiditis (Fig 3). If a fluoroscopically guided LP has been a truly dry tap, repeating the LP under CT guidance can assure definitive needle tip placement.

Before the LP, be sure the footplate is secured at the end of the fluoroscopy table. After the pop into the thecal sac is felt, advance the needle an additional 2-3 mm and then withdraw the stylet to check for return of CSF. If the spinal needle is $25 \mathrm{ga}$, CSF return may be very slow. Flow of CSF can be very unpredictable because even tiny alterations in position can affect flow and the rate of flow. It is critical that once CSF flow is confirmed, great care be taken to maintain the same position of the needle throughout the procedure. The hub can be held with the left hand during attachment of the tubing or manometer. Be careful to not let the syringe or tube torque and rotate the needle.

If CSF flow is confirmed and then stops, reinsert the stylet fully, remove it again, and recheck it. Several maneuvers can help with a dry tap. First, have the patient gently cough or perform a Valsalva maneurver, and this will usually start CSF return. Another step is to raise the fluoroscopy table to about $45^{\circ}$ to fill the distal thecal sac. After several minutes in this position with provocative maneuvers, recheck the flow by removing the stylet. If there is still no flow, with the stylet out, carefully turn the needle $90^{\circ}$ and wait patiently. Continue with this maneuver until a full circle has been achieved. If there is still no flow, the stylet should be replaced and the needle advanced 1-2 $\mathrm{mm}$, followed by a check for CSF return. An additional maneuver is attaching a short tubing and gentle suction with a $10-\mathrm{mL}$ syringe. The suction should be gentle and intermittent, not sustained. Adequately stretch the tubing so that there are few "uphill" kinks through which the CSF has to travel. It may be helpful to hold the tubing lower than the table for gravitational assistance. This is particularly helpful when the table cannot be tilted. In our experience, if CSF flow is initially good, then stops, being patient is most important because usually the flow will start again. Rarely, aspiration is ineffective, and CSF has to be obtained by gravity flow only. This may take up to 30 minutes. The air-leak technique, described previously, can also be attempted.

At this point, a cross-table lateral film if using fluoroscopy or moving the $\mathrm{C}$-arm to confirm the needle position is recommended. If the needle position is confirmed within the canal and there is still no flow, another level can be accessed in the cooperative patient. If a myelography is being performed, $3-5 \mathrm{~mL}$ of myelographic-safe contrast can be instilled to determine whether the needle is intrathecal.

Cases that cannot be successfully accessed with fluoroscopy may require biplane or CT guidance.

If another attempt does not result in CSF, there may be thick 


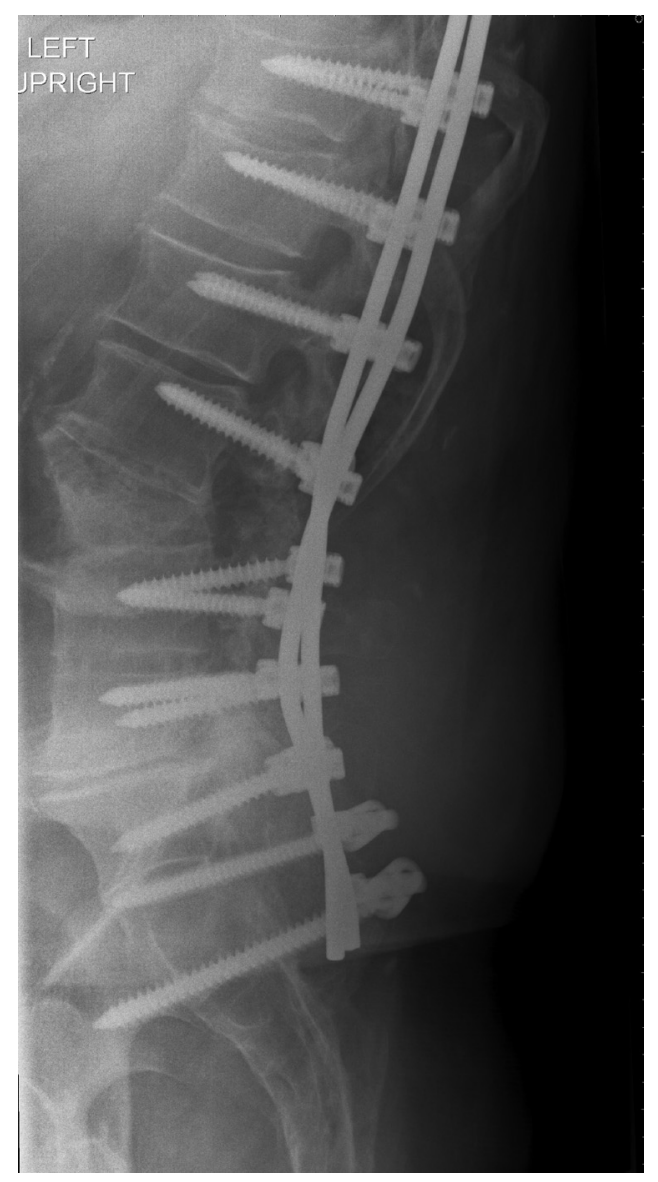

FIG 10. Lateral plain film in a patient with recurrent back pain after bilateral pedicle screws at nearly every level. Note dystrophic ossification from a fusion graft. In a patient with extensive postoperative changes, cross-sectional imaging should be obtained to determine whether there is a patent trajectory for the LP.

tenacious secretions in the CSF as may be seen with meningitis, especially in coccidioidomycosis or arachnoiditis ossificans.

If a second level results in no CSF, then it is truly a dry tap. Our preference is to perform a lumbar spine MR imaging to see whether there is a small thecal sac with a narrowed subarachnoid space, such as in patients with epidural lipomatosis. Depending on the clinical setting and need for CSF, the LP can be attempted another day after hydration or performed with CT guidance or a C1-2 puncture can be considered. Before the C1-2 tap, a cervical spine MR imaging is recommended to be sure there is no cerebellar tonsillar ectopia, which can be present in IIH. Particular attention should be paid to the course of the vertebral and posterior inferior cerebellar arteries. Because of low CSF volume in the subarachnoid space with intracranial hypotension, a dry tap is not uncommon. Additional imaging findings of intracranial hypotension include cerebellar tonsillar ectopia, brain stem slumping, lack of CSF about the orbital optic nerves, dural enhancement, venous engorgement, and cerebral and spinal subdural collections. ${ }^{23-26}$ For patients with intracranial hypotension or a prior dry tap, planning the initial or repeat LP under CT guidance is another consideration. The advantage is immediate confirmation of needle position in the thecal sac.

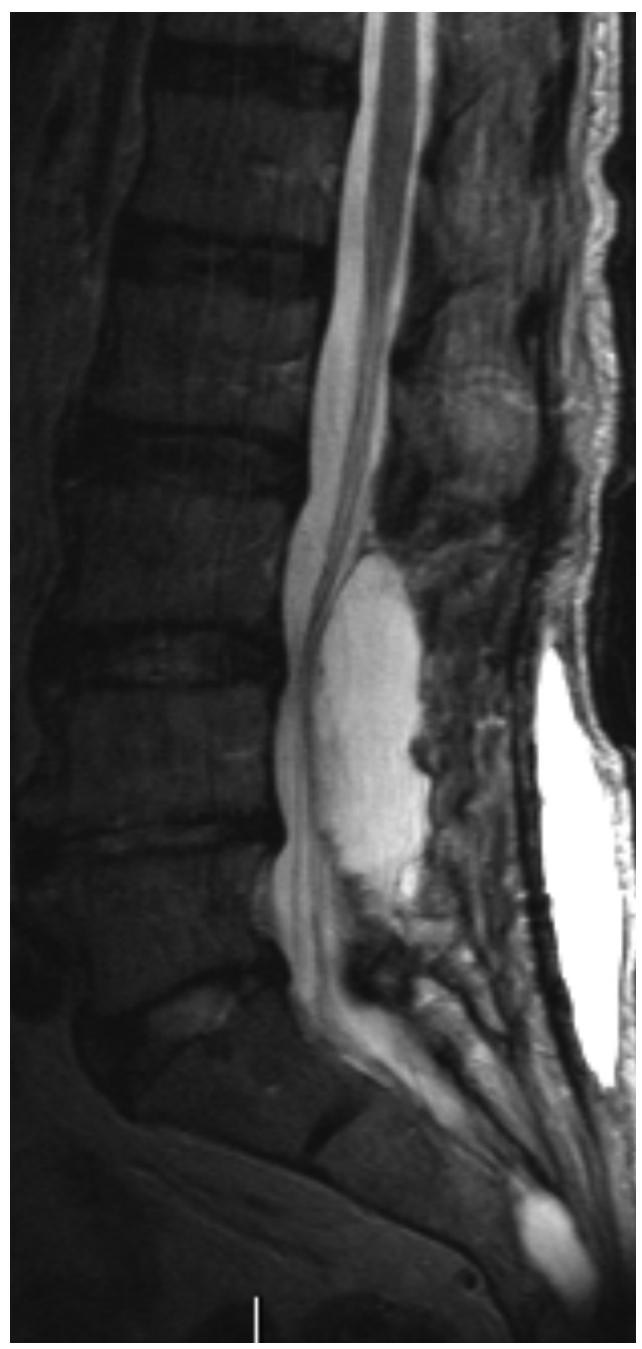

FIG 11. Adult patient with severe back pain following a lumbar spine operation. If LP or myelography is deemed necessary, the needle should be above or below the dorsal intraspinal extradural collection.

\section{The Patient with a Prior Lumbar Spine Surgery}

It is critical that prior imaging be reviewed in a patient with a history of lumbar spine surgery. This review helps avoid bulky osteophytes or osseous fusions; determine whether there is arachnoiditis, which may impair CSF return; and finally, determine the easiest site for the LP (Fig 10). We do not hesitate to go through prior surgical sites. We have not experienced increased pain at prior laminectomy sites, but this experience is patient-dependent. 3D surface-rendered images and models can help the proceduralist visualize the trajectory, especially if there are significant degenerative changes. ${ }^{27}$ In general, a decompressive laminectomy can make the LP easier. However, the procedure can be complicated by arachnoiditis, especially if nerves are adherent to the posterior thecal sac along the course of the needle tract. Postoperative images are also important in determining whether the patient has a dorsal postoperative fluid collection, a seroma, or pseudomeningocele (Fig 11). In general, avoid the fluid collection if you know it is there, to avoid any chance of cross-contamination or confusing imaging or clinical issues with a CSF leak and pseudomeningocele. One of us has inadvertently "sampled" a 


\section{LP dictation template}

The following are included in our LP template:

Reason for LP: [...]

Level of stick, if additional level was attempted: [...]

Fluoroscopy or C-arm: [...]

Coaxial technique vs single needle: [...]

Anesthetic used, amount, bicarbonate used: [...]

Gauge and length of needle: [...]

Opening pressure and position (prone or on side) when pressure

was measured: [...]

Closing pressure (not always performed):

Amount of CSF obtained:

Appearance of CSF:

Symptoms when patient arrived in radiology department:

Resolution of symptoms following LP: [...]

Fluoroscopy time: [...]

Radiation dose: [...]

dorsal seroma in a postoperative patient. If the operation was recent, try to avoid the operative levels, again to prevent infection (or blame for infection) or a confusing picture in a CSF leak. Recent postoperative cases are probably better performed by using CT guidance.

\section{Finally, the Dictation Template}

The obese patient often undergoes serial LPs and knowing what worked previously is helpful to the next proceduralist. We always dictate whether fluoroscopy was adequate or whether the C-arm was necessary. Of course, the level for the successful stick, types of needles used, and whether coaxial technique was needed are included in the report. A general estimate of the necessary needle depth is useful to include in the report. Opening pressure, symptom resolution with the LP, and any other technical facts are included. The anesthetic used, whether bicarbonate was used, and the appearance of the CSF, whether clear, cloudy, or blood-tinged with clearing, are dictated. Finally, fluoroscopy time and radiation dose are always mentioned (Table).

\section{REFERENCES}

1. Kroll H, Duszak R Jr, Nsiah E, et al. Trends in lumbar puncture over 2 decades: a dramatic shift to radiology. AJR Am J Roentgenol 2015; 204:15-19 CrossRef Medline

2. Ogden CL, Carroll MD, Kit BK, et al. Prevalence of childhood and adult obesity in the United States, 2011-2012. JAMA 2014;311: 806-14 CrossRef Medline

3. Hannerz J, Ericson $\mathrm{K}$. The relationship between idiopathic intracranial hypertension and obesity. Headache 2009;49:178-84 CrossRef Medline

4. Mokdad AH, Ford ES, Bowman BA, et al. Prevalence of obesity, diabetes, and obesity-related health risk factors, 2001. JAMA 2003; 289:76-79 Medline

5. Boddu SR, Corey A, Peterson R, et al. Fluoroscopic-guided lumbar puncture: fluoroscopic time and implications of body mass index-a baseline study. AJNR Am J Neuroradiol 2014;35:1475-80 CrossRef Medline

6. Fetterly KA, Schueler BA. Experimental evaluation of fiber-interspaced antiscatter grids for large patient imaging with digital x-ray systems. Phys Med Biol 2007;52:4863-80 CrossRef Medline
7. Bushberg JT. The AAPM/RSNA physics tutorial for residents: X-ray interactions. Radiographics 1998;18:457-68 CrossRef Medline

8. Eskey CJ, Ogilvy CS. Fluoroscopy-guided lumbar puncture: decreased frequency of traumatic tap and implications for the assessment of CT-negative acute subarachnoid hemorrhage. AJNR Am J Neuroradiol 2001;22:571-76 Medline

9. Modica MJ, Kanal KM, Gunn ML. The obese emergency patient: imaging challenges and solutions. Radiographics 2011;31:811-23 CrossRef Medline

10. Brook AD, Burns J, Dauer E, et al. Comparison of CT and fluoroscopic guidance for lumbar puncture in an obese population with prior failed unguided attempt. J Neurointerv Surg 2014;6:324-28 CrossRef Medline

11. Schindera ST, Nelson RC, Toth TL, et al. Effect of patient size on radiation dose for abdominal MDCT with automatic tube current modulation: phantom study. AJR Am J Roentgenol 2008;190: W100-05 CrossRef Medline

12. Yanch JC, Behrman RH, Hendricks MJ, et al. Increased radiation dose to overweight and obese patients from radiographic examinations. Radiology 2009;252:128-39 CrossRef Medline

13. Nayate AP, Nasrallah IM, Schmitt JE, et al. Using body mass index to predict needle length in fluoroscopy-guided lumbar punctures. AJNR Am J Neuroradiol 2016;37:572-78 CrossRef Medline

14. Strupp M, Brandt T, Müller A. Incidence of post-lumbar puncture syndrome reduced by reinserting the stylet: a randomized prospective study of 600 patients. J Neurol 1998;245:589-92 CrossRef Medline

15. Evans RW. Complications of lumbar puncture. Neurol Clin 1998;16: 83-105 CrossRef Medline

16. Wu CL, Rowlingson AJ, Cohen SR, et al. Gender and post-dural puncture headache. Anesthesiology 2006;105:613-18 CrossRef Medline

17. Curry TS III, Dowdey JE, Murry RC Jr. Christensen's Physics of Diagnostic Radiology. 4th ed. Philadelphia: Lea \& Febiger; 1990:258-59

18. Johnson JC, Deeb ZL. Coaxial needle technique for lumbar puncture in the morbidly obese patient. Radiology 1991;179:874 CrossRef Medline

19. Abel AS, Brace JR, McKinney AM, et al. Practice patterns and opening pressure measurements using fluoroscopically guided lumbar puncture. AJNR Am J Neuroradiol 2012;33:823-25 CrossRef Medline

20. Rando TA, Fishman RA. Spontaneous intracranial hypotension: report of two cases and review of the literature. Neurology 1992;42: 481-87 CrossRef Medline

21. Corbett JJ, Mehta MP. Cerebrospinal fluid pressure in normal obese subjects and patients with pseudotumor cerebri. Neurology 1983;33: 1386-88 CrossRef Medline

22. Cauley KA. Fluoroscopically guided lumbar puncture. AJR Am J Roentgenol 2015;205:W442-50 CrossRef Medline

23. Dillon WP, Fishman RA. Some lessons learned about the diagnosis and treatment of spontaneous intracranial hypotension. AJNR Am J Neuroradiol 1998;19:1001-02

24. Mokri B, Piepgras DG, Miller GM. Syndrome of orthostatic headaches and diffuse pachymeningeal gadolinium enhancement. Mayo Clin Proc 1997;72:400-13 CrossRef Medline

25. Schievink WI, Meyer FB, Atkinson JL, et al. Spontaneous spinal cerebrospinal fluid leaks and intracranial hypotension. J Neurosurg 1996;84:598-605 CrossRef Medline

26. Fishman RA, Dillon WP. Dural enhancement and cerebral displacement secondary to intracranial hypotension. Neurology 1993;43: 609-11 CrossRef Medline

27. Cramer J, Quigley E, Hutchins T, et al. Spine Procedures in 3D. Salt Lake City: Practical Imaging; 2016: version 1.1, iTunes Bookstore. https:// itunes.apple.com/us/book/spine-procedures-in-3d/id1119397210?ls= $18 \mathrm{mt}=13$. Accessed February 25, 2017 